\title{
Describing Function Approximation For Biomedical Engineering Applications
}

\author{
Oliver Kinnane $^{\phi}$, John Ringwood ${ }^{\phi}$, Damien Kelly ${ }^{\&}$ and Simon Malpas ${ }^{*}$ \\ ${ }^{\phi}$ Department of Electrical Engineering, \\ NUI Maynooth, \\ Co. Kildare, Ireland \\ E-mail: ${ }^{\phi}$ Oliver.Kinnane@eeng.may.ie \\ E-mail: ${ }^{\phi}$ John.Ringwood@eeng.may.ie \\ ${ }^{\&}$ Department of Electrical Engineering, \\ University College Dublin, \\ Dublin, Ireland \\ E-mail: \&damien.kelly@ucd.ie
}

\begin{abstract}
This paper focuses on the determination of suitable approximations for sigmoid-type nonlinear characteristics, which are common to physiological systems, particularly cardiovascular regulatory systems. These sigmoid nonlinearities have been implicated in the development of limit cycle oscillations in blood pressure. Approximations of the sigmoid are required since the describing function is not calculable for the all representations of the sigmoid characteristic. In this paper, we present a new approximation, which gives a better overall approximation of the sigmoid and hence, can assist the use of describing functions in the diagnostic analysis of cardiovascular function.
\end{abstract}

Keywords - Sigmoid, describing function, cardiovascular function

\section{INTRODUCTION}

It is well established that an oscillation exists at 0.1 $\mathrm{Hz}$ in blood pressure of humans [9]. This oscillation is evident in other species and is shifted to $0.14 \mathrm{~Hz}$ in the dog [1], $0.3 \mathrm{~Hz}$ in the rabbit [7] and $0.4 \mathrm{~Hz}$ in the mouse and rat $[4,6]$. Changes in the strength of this slow oscillation in blood pressure have previously been proposed to reflect changes in the mean level of the sympathetic nerve activity, which changes in heart rate and blood vessel diameter, or changes in the gain of the major regulating feedback loop, the baroreflex. Hence, it is thought that measurement of the strength of the oscillation may be used as a diagnostic measure of neural control of the cardiovascular system in humans [9].

Physiological experimental studies have documented a sigmoid-type nonlinear relationship between blood pressure and sympathetic nerve activity $[3,10]$ in the forward path of the baroreflex. A recent modelling study [12] included this nonlinear characteristic in a feedback loop with a transfer function representing the dynamics of the vasculature system parameterised for the rabbit. They showed that the nonlinearity was involved in the genesis of a limit cycle at the frequency of the oscillation in the blood pressure of the rabbit.

Systems containing nonlinear characteristics can be analysed using describing function techniques. The describing function allows the extension of linear frequency-domain stability analysis techniques to the nonlinear case. The nonlinear element is then represented as a gain term, dependent on the amplitude of the input signal.

Calculation of the describing function for the blood pressure regulating system enables assessment of the presence or absence of a limit cycle oscillation in blood pressure and changes in the strength of this oscillation when the characteristics of the nonlinearity change during different physiological conditions. Hence, an analytical expression for the describing function is required to allow insight into how changes in the parameters of the nonlinearity will effect changes in the strength of limit cycle oscillations in blood pressure. 
Traditional methods of describing function calculation using Fourier series analysis reduce to the problem of calculating certain integrals. For the case of the sigmoid-type nonlinearity a closed-form expression cannot be found for these integrals. A previous method of describing function approximation based on the Taylor series expansion was developed. This method was used by these authors in the prediction of limit cycle oscillations in blood pressure [8] as it enables accurate calculation of the describing function for very low values of input amplitude signal. However, so as to enable accurate calculation of the strength of the lowfrequency blood pressure oscillation and hence, permit the development of a diagnostic measure, a describing function that is accurate for a large range of input amplitude signals is necessary.

This paper presents a more accurate method of approximation of the sigmoid, which enables accurate describing function calculation for a large range of values of the input amplitude signal.

\section{THE DESCRIBING FUNCTION}

The structure of the reduced model of the baroreflex blood pressure regulatory mechanism presented in the study of Ringwood and Malpas [12] is shown in Figure 1.

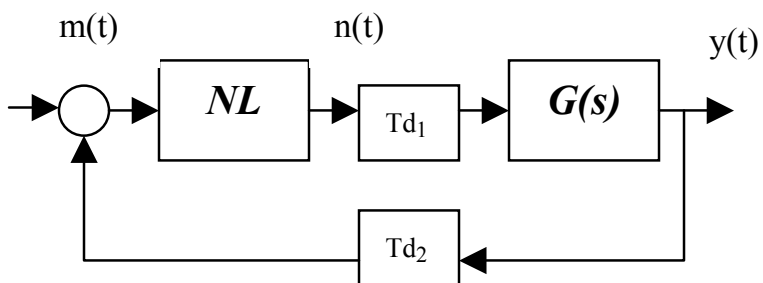

Figure 1: The reduced model of the baroreflex blood pressure controlling mechanism presented by Ringwood and Malpas [12].

where, the dynamics of the vasculature are represented by a first-order transfer function

$$
G(s)=\frac{K}{1+s \tau}
$$

where, $\tau$ is the time constant $(\sim 1.3 \mathrm{~s}) . \mathrm{T}_{\mathrm{d} 1}(\sim 0.6 \mathrm{~s})$ and $\mathrm{T}_{\mathrm{d} 2}(\sim 0.2 \mathrm{~s})$ are the efferent and afferent time delays of the rabbit due to the conduction along the nerves [12].

The nonlinear element, $N L$, in the forward path is the sigmoid, which is shown in Figure 2.

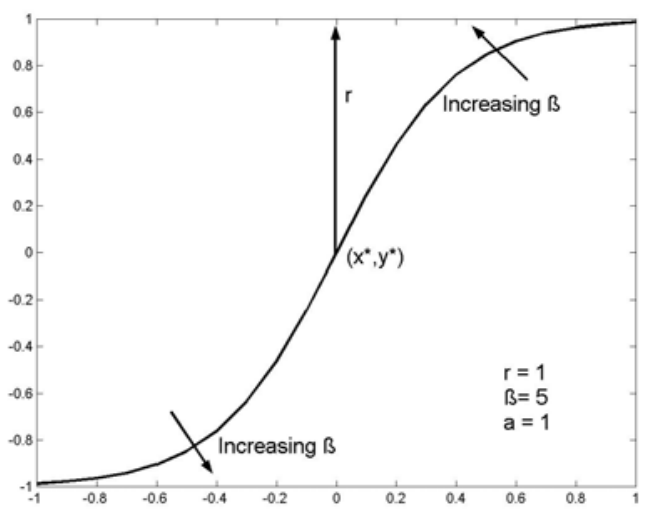

Figure 2: The sigmoid plotted for the parameters given.

The representation used by these authors in previous studies $[8,12]$ to represent the sigmoid is

$$
s(x)=\frac{r}{1+\alpha e^{-\beta\left(x-x^{*}\right)}}-\frac{r}{1+\alpha e^{\beta\left(x-x^{*}\right)}}+y^{*}
$$

Other representations of the sigmoid are common in the physiology literature $[3,10,11]$.

Describing function theory is developed by initially assuming a sinusoidal input to the nonlinear element

$$
M(t)=m \operatorname{Sin}(\omega t)
$$

The output of the nonlinearity may be represented by the Fourier series expansion

$$
N(t)=\frac{A_{0}}{2}+\sum_{k=1}^{\infty} A_{k} \operatorname{Cos}(k \omega t)+\sum_{k=1}^{\infty} B_{k} \operatorname{Sin}(k \omega t)
$$

where,

$$
\begin{aligned}
& A_{k}=\frac{2}{T} \int_{0}^{T} N(t) \operatorname{Cos}(k \omega t) d t \\
& B_{k}=\frac{2}{T} \int_{0}^{T} N(t) \operatorname{Sin}(k \omega t) d t
\end{aligned}
$$

where,

$$
T=\frac{2 \pi}{\omega}
$$

Describing function theory assumes that the transfer function, $G(s)$, low-pass filters the ouput signal of the nonlinearity, hence, all except the fundamental are disregarded.

$$
N(t)=A_{1} \operatorname{Cos}(\omega t)+B_{1} \operatorname{Sin}(\omega t)
$$

The describing function is defined as

$$
N(m, \omega)=\frac{B_{1}+j A_{1}}{m}
$$

The sigmoid is an odd function when centred at the origin, therefore, $A_{1}=0$ 
and,

$$
B_{1}=\frac{1}{\pi} \int_{-\pi}^{\pi}\left[\frac{r \operatorname{Sin}(x)}{1+e^{-\beta\left(m \operatorname{Sin}(x)-x^{*}\right)}}-\frac{r \operatorname{Sin}(x)}{1+e^{\beta\left(m \operatorname{Sin}(x)-x^{*}\right)}}+y^{*}\right] d x
$$

This integral may be calculated using numerical integration methods. However, no closed-form analytic expressions exist for it. Similarly, no closedform expressions exist for the other representations of the sigmoid [10, 11]. Hence, traditional methods of describing function analysis are not applicable for this example, if a closed-form expression is required. An alternative representation of the sigmoid must be found which avoids the occurrence of this problem.

\section{DESCRIBING FUNCTION APPROXIMATIONS}

a) Describing function approximation using the Taylor series expansion of the sigmoid.

Holohan introduced a method of describing function approximation based on the Taylor series expansion of the sigmoid [5]. The approximation of the sigmoid using 6 terms of the Taylor series expanded around the origin is shown in Figure 2. The sigmoid is described for the parameters listed in Figure 1.

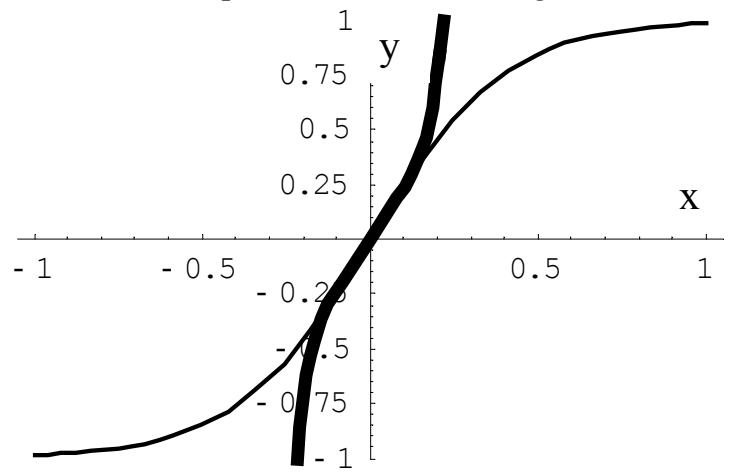

Figure 3: The sigmoid (thin line) and the approximation of the sigmoid for 6 terms of the Taylor series expansion (thick line).

Increasing the number of terms of the Taylor series expansion increases the accuracy of the approximation around the origin but no increase in the accuracy outside of this region is attained.

When the input to the nonlinearity $s(x)$ is $m \operatorname{Cos}(\omega t)$, the output is $s(m \operatorname{Cos}(\omega t))$. What results for finite number of terms of the Taylor series expansion is an approximation to the Fourier series expansion of $s(m \operatorname{Cos}(\omega t))$. As in equation 9, the describing function $N(m)$ is then the coefficient of the fundamental divided by the amplitude of the input signal.
Referring to the block diagram of Figure 1, and replacing the nonlinear function $s(x)$ by its describing function representation, $N(m)$, a linear systems approach produces the characteristic equation

$$
1+N(m) G(j \omega)=0
$$

or,

$$
G(j \omega)=-\frac{1}{N(m)}
$$

When considering a specific value of input amplitude $(m)$, the Nyquist stability criterion is easily adapted to the nonlinear system analysis situation by considering encirclements of the point $-1 / N(m)$ in the $G(j \omega)$ plane.

This well-known theory has already been used by these authors with success when predicting the possible occurrence of limit cycle oscillations in blood pressure [8]. By setting the input amplitude to zero the possible existence of a limit cycle oscillation was investigated for a range of physiological conditions.

However, when considering all oscillation amplitudes $(0<m<\infty)$, the representation of $-1 / N(m)$ generates a locus in the $G(j \omega)$ plane. If the $G(j \omega)$ contour in the complex plane intersects the $-1 / N(m)$ locus, the intersection satisfies the condition for a sustained limit cycle oscillation. The intersection point defines values of frequency (from $G(j \omega)$ ) and amplitude (from $-1 / N(m)$ ) for this limit cycle oscillation.

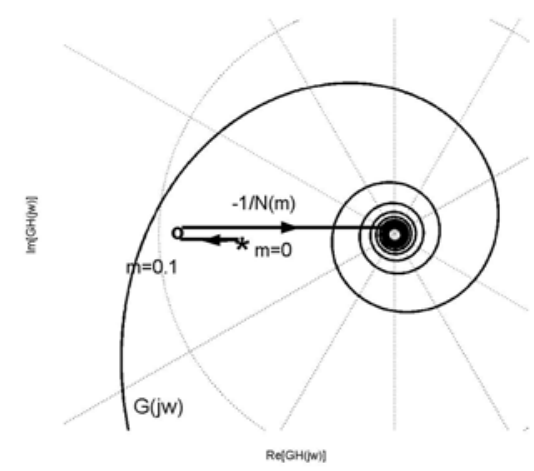

Figure 4: Nyquist plot showing the $G(j w)$ curve given by equation 1 and the $-1 / N(m)$ locus developed using the Taylor series expansion method. 
Using the Taylor series expansion method of sigmoid approximation the $-1 / N(m)$ curve produces erroneous results outside the range of accuracy of the sigmoid approximation. Outside this range the $-1 / N(m)$ curve doubles back and increases in the opposite direction for increasing values of the input amplitude signal, $m$.

Hence, the resulting values of amplitude and frequency of the limit cycle oscillation are not correct for a large range of input amplitude values.

Considering this problem an alternative method of representing the sigmoid was considered. A function approximation method based on orthogonal polynomial expansion was developed.

b) Describing function approximation using Legendre polynomial approximation of the sigmoid

Let $w$ be a positive function on $[a, b] \subset \square$.

Let

$$
L^{2}([a, b], w)=\left\{f:[a, b] \rightarrow \square: \int_{a}^{b}|f(x)|^{2} w(x) d x<\infty\right\}
$$

$L^{2}([a, b], w)$ is an inner product space [2] with inner product

$$
\langle f \mid g\rangle=\int_{a}^{b} f(x) \bar{g}(x) w(x) d x
$$

Let $\left\{e_{n}\right\}$ be an orthonormal basis for $L^{2}([a, b], w)$. Let $U_{N}$ be the finite dimensional subspace of $L^{2}([a, b], w)$ spanned by $\left\{e_{1}, \ldots, e_{N}\right\}$.

Then, given $f \in L^{2}([a, b], w)$ the element $\hat{f} \in U_{n}$ such that $\int_{a}^{b}|f(x)-\hat{f}(x)|^{2} w(x) d x$ is minimised, is the orthogonal projection $\hat{f}=P_{U_{N}} f=\sum_{n=1}^{N}\left\langle f, e_{n}\right\rangle e_{n}$ of $f$ onto $U_{N}$.

Let $U_{6}$ be the dimensional subspace of $L^{2}[-1,1]$ spanned by the first 6 Legendre polynomials.

$$
\begin{aligned}
& e_{1}(x)=\frac{1}{\sqrt{2}} \\
& e_{2}(x)=\sqrt{3 / 2} x \\
& e_{3}(x)=\sqrt{15 / 14}\left(2 x^{2}+1\right) \\
& e_{4}(x)=\sqrt{35 / 34}\left(4 x^{3}-3 x\right) \\
& e_{5}(x)=3 \sqrt{7 / 62}\left(1-8 x^{2}+8 x^{4}\right) \\
& e_{6}(x)=3 / 7 \sqrt{11 / 2}\left(5 x-20 x^{3}+16 x^{5}\right)
\end{aligned}
$$

$s(x) \in L^{2}[-1,1]$, the function defined in equation (2), is approximated by

$$
P_{u} s=\sum_{n=1}^{6}\left\langle s \mid e_{n}\right\rangle e_{n} \in U
$$

with minimal $L^{2}[-1,1]$ error

i.e. $\int_{a}^{b}\left|s(x)-P_{u} s(x)\right|^{2} d x=\min _{g \in U} \int_{a}^{b}|s(x)-\hat{s}(x)|^{2} d x$

Functions can be approximated over different intervals than $[-1,1]$ through dilation and normalisation of the orthonormal basis and the accuracy of the approximation can be increased by increasing the number of Legendre polynomials used.

Other sets of orthogonal polynomials including Chebyshev polynomials of the first kind where $w(x)=\left(1-x^{2}\right)^{-1 / 2}$ and Chebyshev polynomials of the second-kind where $w(x)=\sqrt{1-x^{2}}$ were also investigated, but, the Legendre polynomials gave the best approximation.

The sigmoid $s(x)$ and the approximation to the sigmoid $\hat{s}(x)$ are plotted in Figure 6 for 6 terms of Legendre polynomials.

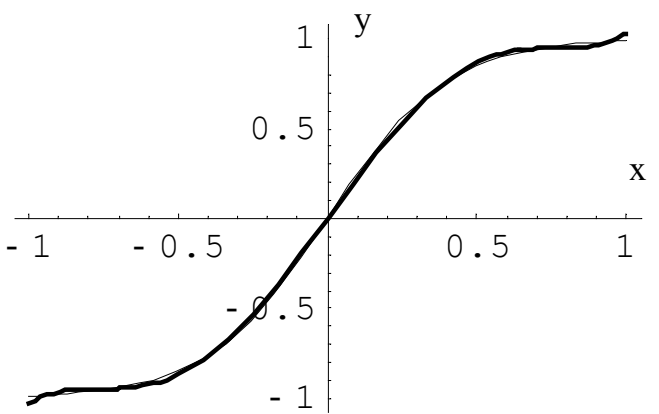

Figure 5: The sigmoid (thin line) and the approximation of the sigmoid for 6 terms of the Legendre polynomial expansion (thick line). 
The describing function may be developed in the same way as already documented for the Taylor series expansion method. Hence, resulting in a series that resembles a Fourier trigonometric series.

Using this method of approximation, reliable values of amplitude and frequency for a large range of input amplitude values can be calculated from the intersection of the $G(j \omega)$ curve and the $-1 / N(m)$ curve. This is shown in Figure 6.

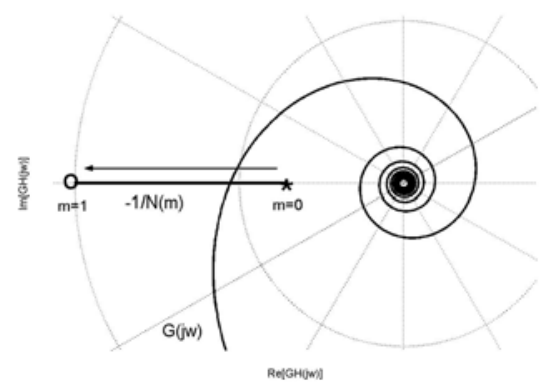

Figure 6: Nyquist plot showing the $G(j w)$ curve given by equation 1 and the $-1 / N(m)$ locus developed utilising the Legendre polynomials.

\section{COMPARISON OF THE DESCRIBING FUNCTION APPROXIMATION METHODS}

The describing function gains calculated for the sigmoid parameters given in Figure 1 and for a range of input amplitude values are shown in Figure 7. The describing function gains calculated utilising the Taylor series expansion and the Legendre polynomials, and the true value of the describing function given by numerical integration methods are compared.

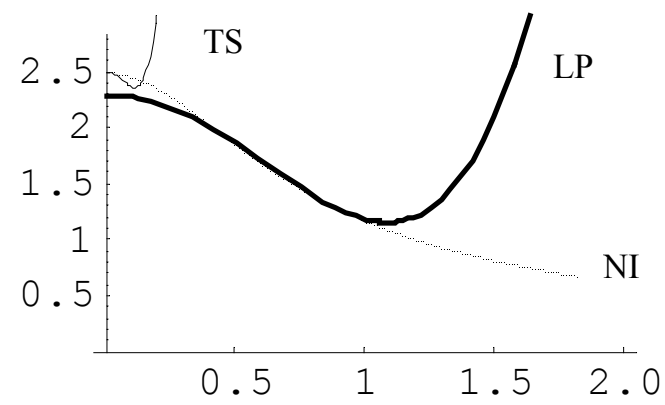

$\mathrm{m}$

Figure 7: The describing function gain $(\mathrm{N}(\mathrm{m}))$ for the Taylor series approximation method (TS), the Legendre polynomial approximation method (LP) and the true gain as calculated using numerical integration (NI) plotted against increasing amplitude of the input signal (m).

The difference between the describing function approximation methods and the describing function calculated using numerical integration are compared in Figure 8.

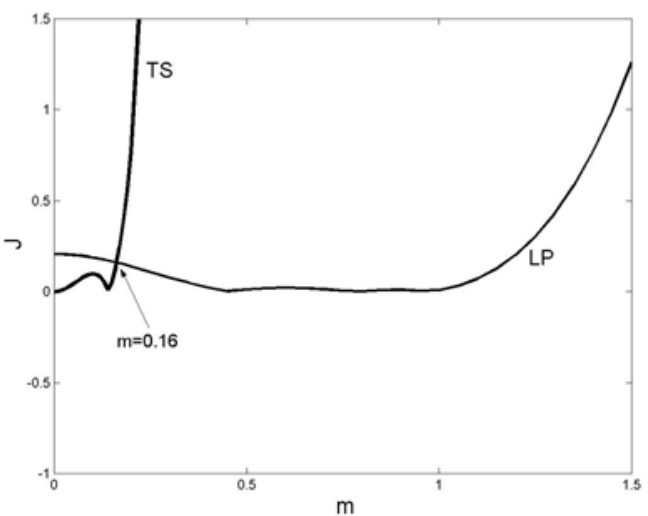

Figure 8: The difference, J, between the describing function calculated using numerical integration and those calculated using the Taylor series expansion (TS) and the method utilising Legendre polynomials (LP).

The describing function method utilising the Taylor series expansion is more accurate for values of input amplitude up to 0.16 , after this the approximation based on the Legendre polynomials becomes more accurate. Outside the range of approximation of the sigmoid the describing function method based on the Legendre polynomials loses accuracy.

The methods of describing function approximation may also be analysed by comparing the predicted amplitude of the limit cycle with that of a simulated example in a limit cycle condition. A model with the structure of that shown in figure 1 was used with results documented in Table 1 . The gain of the dynamic transfer function was varied so as to alter the amplitude of the limit cycle.

\begin{tabular}{|c|c|c|c|}
\hline $\boldsymbol{K}$ & $\begin{array}{c}\boldsymbol{m} \\
\text { Simulated }\end{array}$ & $\begin{array}{c}\text { Predicted } \\
\boldsymbol{m} \boldsymbol{T S}\end{array}$ & $\begin{array}{c}\text { Predicted } \\
\boldsymbol{m} \text { OP }\end{array}$ \\
\hline 1.2 & 0 & 0 & 0 \\
\hline 1.3 & 0.08 & 0.03 & 0 \\
\hline 1.4 & 0.238 & 0 & 0 \\
\hline 1.5 & 0.33 & 0 & 0.27 \\
\hline 1.6 & 0.41 & 0 & 0.395 \\
\hline 1.7 & 0.48 & 0 & 0.47 \\
\hline 2 & 0.65 & 0 & 0.658 \\
\hline 2.2 & 0.76 & 0 & 0.75 \\
\hline 2.5 & 0.915 & 0 & 0.88 \\
\hline 3 & 1.15 & 0 & 0 \\
\hline
\end{tabular}

Table 1: Simulated results of limit cycle oscillation amplitude calculated for increasing values of $\mathrm{K}$ and predicted values of this amplitude using the 2 approximation methods.

In Table 1, 0 implies no limit cycle. The Legendre polynomial method of describing function approximation allows for good prediction of the limit cycle oscillation for a large range of amplitude values. However, the Taylor series expansion 
method allows better prediction of the amplitude of the limit cycle oscillation for small amplitude values. Hence, the Taylor series expansion offers a better approximation of the sigmoid around the inflection region for equivalent number of terms of both expansion methods. The inflection sets up the limit cycle oscillation. As a result the describing function calculated using the Taylor series expansion method allows for better detection of the presence or absence of the oscillation.

However, outside the inflection region the approximation method based on the Legendre orthogonal polynomial expanion method is much better at approximating the sigmoid nonlinearity. The values of the describing function for input amplitude signals up to the full input range of the sigmoid, can be accurately approximated when compared with the results of the simulated example and those of numerical integration.

\section{CONCLUSIONS}

This paper presents an approximation to the sigmoid nonlinearity that is common in physiological systems. This approximation enables calculation of a closed-form expression for the describing function. Unlike the previous approximation method, utilising the Taylor series, the method of approximation presented in this paper allow an accurate calculation of the describing function for a large range of input amplitude. This enables calculation of the amplitude of the limit cycle oscillation.

Of the two methods of approximation used in this study, the method based on the Taylor series expansion is better for detection of a limit cycle, while the method of approximation based on the orthogonal polynomials permits accurate calculation of the amplitude of the oscillation.

These results are significant when applied to the field of biomedical engineering and particularly the area of cardiovascular control where the strength of oscillations in blood pressure are proposed as a future diagnostic test [9]. The development of an accurate closed-form expression for the describing function of the signoid allows greater insight into how changes in the parameters of the nonlinearity will effect the strength of these limit cycles oscillations in blood pressure.

\section{ACKNOWLEDGEMENTS}

We wish to thank Dr. Bernard Kelville and Dr. Sean McLoone for their help and generosity with their time.

\section{REFERENCES}

[1] Akselrod SD, Gordon D, Madwed JB, Snidman NC, Shannon DC, and Cohen RJ. Power spectrum analysis of heart rate fluctuations: a quantitative probe of beat-tobeat cardiovascular control. Science 213: 220-222, 1981.

[2] Barrett C, Ramchandra, R, Guild, SJ, Lala, A, Budgett, DM and Malpas, SC. What sets the long-term level of renal sympathetic nerve activity, a role for angiotensin II and baroreflexes? Circ. Res. 92: 1330-1336, 2003.

[3] Brown DR, Brown LV, Patwardhan A, and Randall DC. Sympathetic activity and blood pressure are tightly coupled at $0.4 \mathrm{~Hz}$ in conscious rats. Am. J. Physiol. 267: R1378R1384, 1994.

[4] Holohan A. On calculating the describing function. In: Irish signals and systems conference, Dublin, 2000.

[5] Janssen BJA, Leenders PJA, and Smits JFM. Short-term and long-term blood pressure and heart rate variability in the mouse. American Journal of Physiology Regulatory Integrative \& Comparative Physiology 278(1): R215-R225, 2000.

[6] Janssen BJA, Malpas SC, Burke SL, and Head GA. Frequency-dependent modulation of renal blood flow by renal nerve activity in conscious rabbits. Am J Physiol 273(2): R597-608, 1997.

[7] Kinnane OP, Ringwood, J.V. and Malpas, S.C. Predicting the slow oscillation in blood pressure using nonlinear analysis. In press, 2004.

[8] Malliani A, Pagani M, Lombardi F, and Cerutti S. Cardiovascular neural regulation explored in the frequency domain. Circulation 84(2): 482-92, 1991.

[9] Malpas SC, Bendle RD, Head GA, and Ricketts JH. Frequency and amplitude of sympathetic discharges by baroreflexes during hypoxia in conscious rabbits. $\mathrm{Am} \mathrm{J}$ Physiol 271(6): H2563-H2574, 1996.

[10] Ricketts JH and Head GA. A five-parameter logistic equation for investigating asymmetry of curvature in baroreflex studies. American Journal of Physiology Regulatory Integrative \& Comparative Physiology 46(2): R441-R454, 1999.

[11] Ringwood JV and Malpas SC. Slow oscillations in blood pressure via a nonlinear feedback model. Am. J. Physiol. 280(4): R1105-984, 2001. 